\title{
Molecular and cellular heterogeneity: the hallmark of glioblastoma
}

\author{
Diane J. Aum, B.S., ${ }^{1}$ David H. Kim, M.S., ${ }^{1}$ Thomas L. Beaumont, M.D., Ph.D., ${ }^{1}$ \\ Eric C. Leuthardt, M.D., ${ }^{1,6-9}$ Gavin P. Dunn, M.D., Ph.D., 1,4,5,7 \\ and Albert H. Kim, M.D., Ph.D. ${ }^{1-3,6,7}$ \\ Departments of ${ }^{1}$ Neurological Surgery, ${ }^{2}$ Neurology, ${ }^{3}$ Developmental Biology, ${ }^{4}$ Pathology and Immunology, \\ ${ }^{8}$ Biomedical Engineering, and ${ }^{9}$ Mechanical Engineering and Material Sciences; ${ }^{5}$ Center for Human \\ Immunology and Immunotherapy Programs; ${ }^{6}$ Center for Innovation in Neuroscience and Technology; and \\ ${ }^{7}$ Siteman Cancer Center, Washington University School of Medicine in St. Louis, Missouri
}

\begin{abstract}
There has been increasing awareness that glioblastoma, which may seem histopathologically similar across many tumors, actually represents a group of molecularly distinct tumors. Emerging evidence suggests that cells even within the same tumor exhibit wide-ranging molecular diversity. Parallel to the discoveries of molecular heterogeneity among tumors and their individual cells, intense investigation of the cellular biology of glioblastoma has revealed that not all cancer cells within a given tumor behave the same. The identification of a subpopulation of brain tumor cells termed "glioblastoma cancer stem cells" or "tumor-initiating cells" has implications for the management of glioblastoma. This focused review will therefore summarize emerging concepts on the molecular and cellular heterogeneity of glioblastoma and emphasize that we should begin to consider each individual glioblastoma to be an ensemble of molecularly distinct subclones that reflect a spectrum of dynamic cell states.
\end{abstract}

(http://thejns.org/doi/abs/10.3171/2014.9.FOCUS14521)

\section{KeY WORDS • glioblastoma $\bullet$ glioma $\bullet$ genomic $\bullet$ heterogeneity $\bullet$ IDH1}

$\mathrm{G}$ LIOBLASTOMA is the most common primary malignant brain tumor in adults and continues to portend poor prognosis despite decades of research. Even with aggressive resection followed by concomitant chemotherapy and radiation, there is a high recurrence rate with median survival of less than 15 months. ${ }^{58}$ Indeed, fewer than $5 \%$ of patients survive 5 years. ${ }^{45}$ Extensive investigation of the cellular and molecular biology of glioblastoma over the last decade has identified several histopathological and chromosomal hallmarks that have enhanced diagnosis and clinical stratification without a significant improvement in overall outcome. Nevertheless, a deepening focus on the molecular characteristics of glioblastoma has revealed that this histopathological term comprises a range of diseases, some of which have divergent natural histories. Indeed, it is becoming clear that malignant glioma exhibits striking cellular and molecular heterogeneity not only across but also within glioblastomas. In fact, a patient with glioblastoma likely

\footnotetext{
Abbreviations used in this paper: G-CIMP $=$ glioma $\mathrm{CpG}$ island methylator phenotype; H3K27Ac = histone H3 Lysine27 acetylation; RTK = receptor tyrosine kinase; $\mathrm{SCNA}=$ somatic copy number alteration; TCGA $=$ The Cancer Genome Atlas; TIC = tumor-initiating cell; $2-\mathrm{HG}=2$-hydroxyglutarate.
}

harbors several different diseases within the same tumor such that each tumor is actually a complex and dynamic constellation of cellular and molecular changes that contribute to disease pathobiology.

Cellular and molecular heterogeneity is not unique to glioblastoma and has been extensively studied in several other solid tumors. Next-generation sequencing of subregions and even single cells within breast, ${ }^{42}$ pancreas, ${ }^{12,68}$ prostate, ${ }^{27}$ and renal ${ }^{25}$ cancer have demonstrated extensive regional clonality within primary tumors, where only one-third of mutations, known as founding mutations, occur ubiquitously in all cells. Remarkably, even minor subclones from a primary tumor may become treatmentrefractory clones that dominate the recurrence. ${ }^{27}$ This concept of clonal evolution has been firmly established in acute myeloid leukemia, where deep sequencing has revealed that subclones representing as little as $5 \%$ of the total tumor cells can survive treatment, acquire new mutations, and lead to relapse. ${ }^{21}$ In the related context of metastatic disease, deep sequencing of driver mutations identified in genomic studies of medulloblastoma revealed that while distinct metastatic clones within a given patient are genetically similar, they are divergent from the primary tumor, suggesting subclonal enrichment and/or evolution. ${ }^{4,66}$ 
It is clear from these studies in other cancers and a quickly accumulating body of literature in glioblastoma that an improved understanding of glioblastoma heterogeneity has important implications not only for the direction of future scientific investigation, but also for clinical diagnosis and patient management. Herein, we review the salient themes of tumor heterogeneity on the molecular and cellular levels and highlight the critical studies germane to neurosurgeons and neuro-oncologists that are driving our growing understanding that diversity and heterogeneity in malignant glioma are the rules, rather than the exceptions (Table 1).

\section{Molecular Heterogeneity}

\section{Molecular Classification: Diversity Across Tumors at the RNA Level}

Transcriptomic Subtypes. Commitment by cancer biologists to understanding the genomic basis of glioblastoma has yielded ever higher-resolution pictures of the types of alterations that glioblastomas harbor. These studies have been central to the ongoing revolution in cancer genomics that is revealing the molecular origins of every cancer type and establishing a new disease taxonomy founded on shared and/or recurrent genomic lesions. ${ }^{40}$ Broadly speaking, this sea change in cancer genomics has been possible due to our ability to perform genome-wide profiling of tumor DNA and RNA in a systematic fashion. ${ }^{23}$ In the case of glioblastoma, perhaps one of the greatest influences in our early understanding of glioma heterogeneity was the application of transcriptional profiling to brain tumor classification. ${ }^{10,30}$ Transcriptional profiling, pioneered by Golub et al. ${ }^{26}$ and Alizadeh and colleagues, ${ }^{1}$ has provided powerful evidence demonstrating the striking heterogeneity that exists among glioblastomas arising in different patients. Among many, 2 studies in particular have provided our working foundation of glioblastoma subtypes defined by transcriptomic structure. Phillips et al. identified 3 transcriptional subtypes-proneural, mesenchymal, and proliferativebased on expressed genes that were most strongly correlated with survival. ${ }^{49}$ Complementing this work, Verhaak et al. employed unsupervised clustering approaches to classify 200 glioblastomas into 4 subtypes-proneural, mesenchymal, classical, and neural ${ }^{63}$ As discussed below, these subtypes also harbor distinct DNA alterations, further emphasizing intertumoral heterogeneity and distinct pathways of tumor evolution. Importantly, although the specific number of subtypes defined by these 2 studies differs, the proneural and mesenchymal classifications appear to be the most robust and concordant. ${ }^{30}$ Specifically, both studies identified expression of proneural genes such as DLL3 and OLIG2 as well as mesenchymal class expression of CD40 and CHI3L1/YKL-40. Further work has attempted to develop prognostic assays based on a limited set of genes that correlates with survival, which is compelling but has not yet yielded completely overlapping gene sets across studies., ${ }^{9,19}$ Nevertheless, the study of gene expression programs and signatures has been pivotal to the evolution of a conceptual framework that glioblas- toma development is variable from patient to patient-i.e., the "subtype" heuristic - and surely will continue to drive key biological insights. Although beyond the scope of this review, there has also been a surge of interest in the epigenetic landscape of glioblastoma, and further understanding of these structural epigenetic phenotypes will likely yield similarly important insights into intertumoral heterogeneity and natural history. ${ }^{11,59}$

\section{Molecular Classification: Diversity Across Tumors at the DNA Level}

The landmark glioblastoma expression mRNA profiling studies have been complemented by equally pivotal DNA profiling studies. To date, the glioblastoma genome is one of the most comprehensively annotated among all cancer types, and the extensive work in this area has highlighted many of the recurrent pathways that are altered in these tumors. At the DNA level, glioblastoma is similar to other cancers in that there are gains and losses of gene regions and even whole chromosomes; these somatic copy number alterations (SCNAs) have been cataloged by a number of groups ${ }^{6,48,52,65}$ using sophisticated computational methodologies such as Genomic Identification of Significant Targets in Cancer (GISTIC) ${ }^{6,41}$ and Genomic Topography Scan. ${ }^{65}$ Together, these studies identified commonly amplified genes such as EGFR, MET, PDG$F R A, M D M 2, P I K 3 C A, C D K 4$, and $C D K 6$ as commonly deleted genes including $C D K N 2 A / B, P T E N$, and $R B 1$.

In addition to clarifying the gains and losses of DNA segments, more recent work has focused on the alterations in glioblastoma at the single nucleotide level using sequencing approaches. There is no question that the application of next-generation sequencing approaches to the study of cancer has fueled a punctuated leap in our understanding of the genomic landscape across all cancer types. Work from the Vogelstein group ${ }^{46}$ and The Cancer Genome Atlas (TCGA) $)^{13}$ first demonstrated the types and frequencies of mutations seen in glioma, albeit using distinct approaches. While both provided significantly overlapping information, Parsons et al. ${ }^{46}$ identified recurrent mutations in the IDHI gene, which was a seismic finding to be discussed further below, while work from the TCGA paper also provided a proof-of-principle showing that systematic, multiplatform profiling of tumor types was possible. These studies were a prelude to the comprehensive, nearly encyclopedic anthology of the glioblastoma genome published by TCGA working group in 2013. ${ }^{11}$ This study, analyzing more than 500 glioblastomas, integrated information from sequencing, SCNA analysis, transcriptomic analysis, epigenetic profiling, and rearrangement studies to provide a breathtaking molecular picture of these tumors. Recurrent mutations were identified in genes such as PTEN (31\%), TP53 (29\%), EGFR (26\%), PIK3R1 (11\%), PIK3CA (11\%), and IDHI (5\%). In addition, TERT promoter mutations were identified in the majority of tumors assessed by whole genome sequencing, corroborating previous findings. ${ }^{34}$ Novel mutations in genes such as LZTR, SPTA1, and TCHH were also identified.

Most importantly, the systematic TCGA profiling of a large number of tumors enabled high-level integration of structural genomic information that provided a more 
Molecular and cellular heterogeneity in glioblastoma

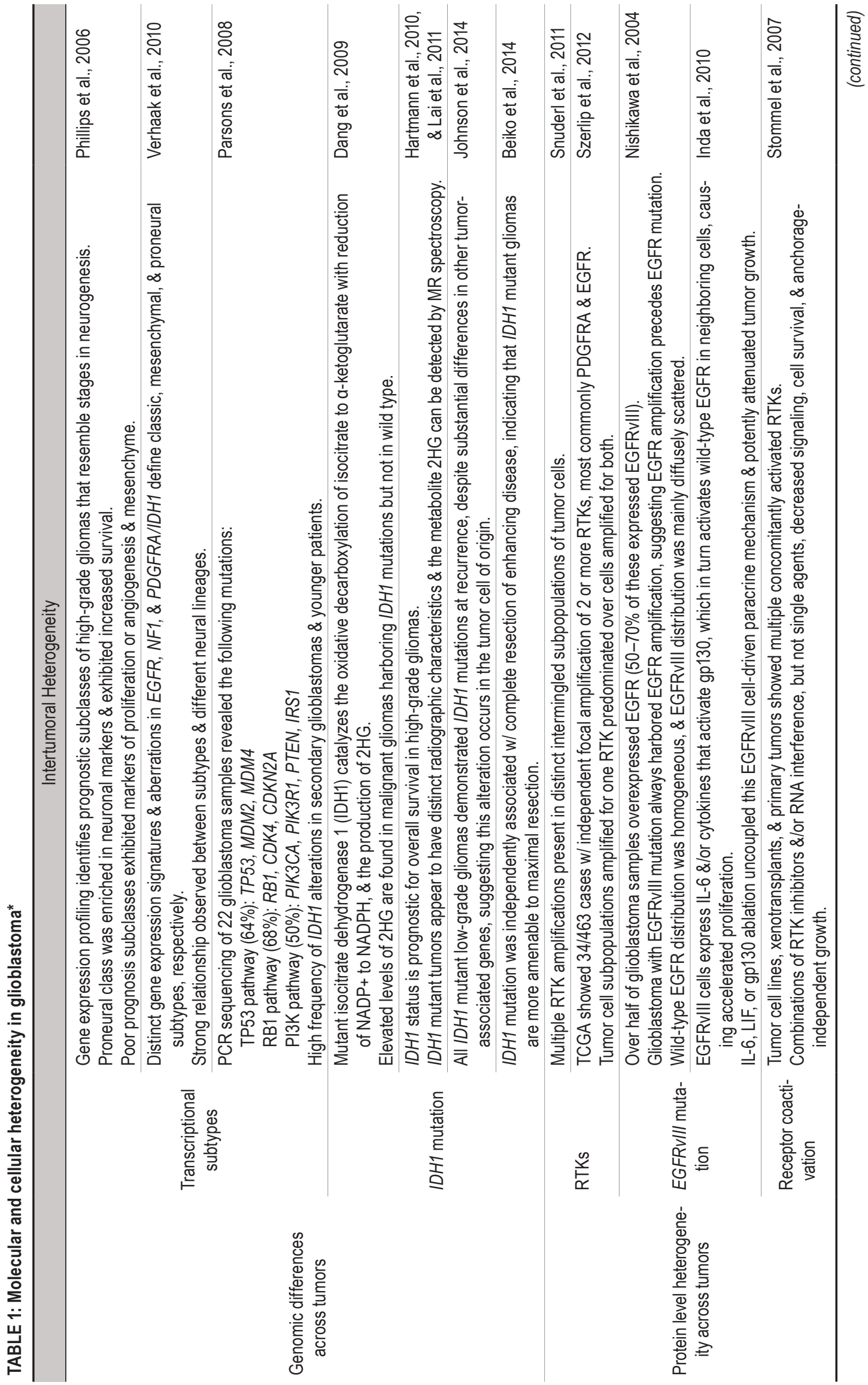




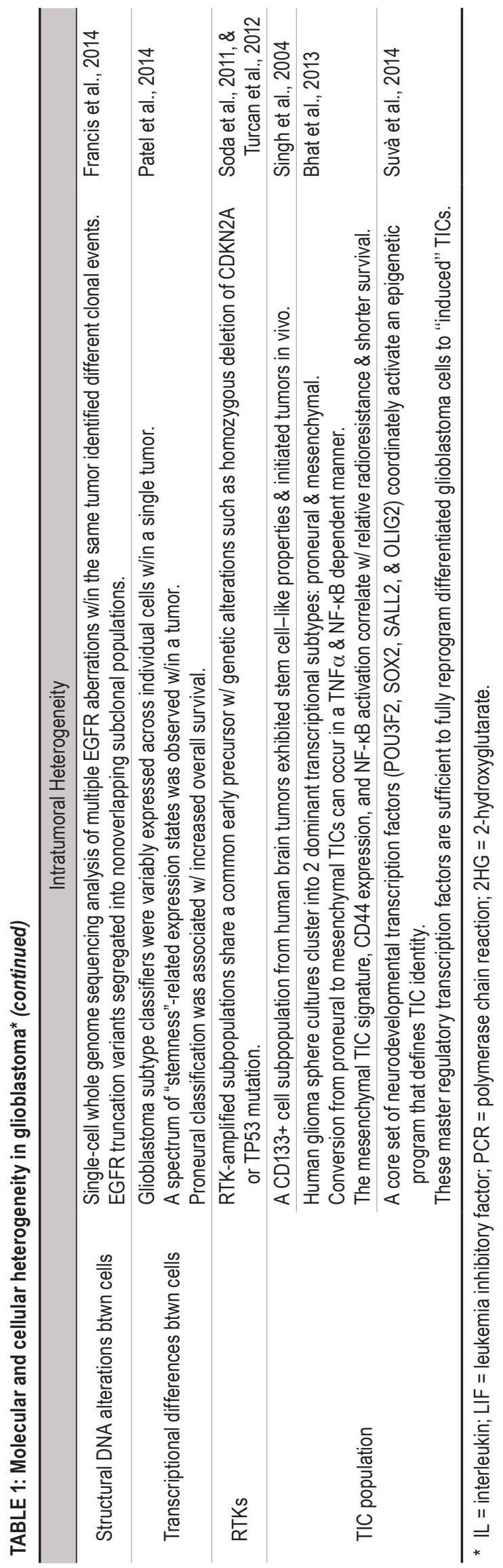

granular picture of intertumor heterogeneity. Specifically, it was possible to integrate DNA-based alterations with RNA-defined subtypes. For instance, proneural tumors often harbor mutations in IDH1, TP53, and ATRX and can exhibit the glioma $\mathrm{CpG}$ island methylator phenotype (G-CIMP); IDH1 wild-type tumors tend to be G-CIMP negative and harbor PDGFRA/CDK4 amplifications. In contrast, mesenchymal tumors are $I D H I$ wild type but harbor NF1 mutations. In addition, classical tumors more frequently harbor $E G F R$ mutations, including the EGFR$v I I I$ intragenic deletion, in the setting of high-level EGFR amplification. Additional work in this effort provided information on novel gene rearrangements and epigenetic landscapes as well. Taken together, this study provided high-resolution analysis of the molecular diversity observed across different glioblastoma tumors. This information can be accessed either through TCGA (http://cancergenome.nih.gov) or through independent portals that enable rapid accession of the data in visual format (Broad Institute TCGA portal at http://www. broadinstitute.org/ tcga and the Memorial Sloan-Kettering Cancer Center cBio Portal at http://www.cbioportal.org).

\section{The IDH1 Story: Not all Gliomas Are Created Equal}

If there is a single mutation that underscores the idea that not all glioblastomas are created equally, it is the mutation found in the IDHI gene. ${ }^{22,23}$ IDHI encodes isocitrate dehydrogenase 1 , a cytoplasmic enzyme that catalyzes the oxidative decarboxylation of isocitrate to $\alpha$-ketoglutarate and NAD+ to NADPH. ${ }^{50}$ Recurrent mutations in $I D H 1$ were first identified by Parsons et al. in 2008 in a study in which 5 of 22 tumors in the study's discovery set harbored a single mutation encoding the IDH1 $\mathrm{R} 132 \mathrm{H}$ mutant protein. Extensive work by many groups has since demonstrated how important the distinction between "IDH1 mutant" and "IDHI wild type" may be. In brain tumors, IDH1 mutations are typically $\mathrm{R} 132 \mathrm{H}$ (90\%), although other substitutions at position R132 are also found. ${ }^{69} \mathrm{IDH} 1$ mutations are identified in $60 \%-80 \%$ of Grade II and III astrocytomas, oligodendrogliomas, and oligoastrocytomas. ${ }^{23,28,29}$ Whereas $5 \%-6 \%$ of primary glioblastomas harbor IDH1 mutations, the majority of secondary glioblastomas carry this mutation. Similar mutations in the IDH2 gene often seen in acute myeloid leukemia are rarely found in glioma. Interestingly, mutations in the $I D H$ genes have also been identified in cholangiocarcinomas and cartilaginous tumors. ${ }^{23}$

The foundation of all efforts to understand $I D H 1 \mathrm{mu}-$ tant protein biology is the work of Dang and colleagues. ${ }^{20}$ This study used a liquid chromatography/mass spectrometry metabolomics approach to discover that IDH1$\mathrm{R} 132 \mathrm{H}$ mutant proteins acquire a neomorphic function that leads to the production of high levels of the " $R$ " enantiomer of 2-hydroxyglutarate (2-HG) that are never seen at comparable levels in an IDHI wild-type setting and can be detected using MR spectroscopy. ${ }^{2}$ Because tumor cells typically harbor one copy of IDHI mutation and do not exhibit loss of heterozygosity at that locus, these mutations are likely gain of function in nature. Significant effort is underway to understand the mechanistic underpinnings of the IDH1 mutant state. Although 2-HG 
has been shown to inhibit the $\alpha$-ketoglutarate-dependent superfamily of dioxygenases affecting histone and DNA methylation, ${ }^{18,67}$ likely contributes to the G-CIMP phenotype, ${ }^{62}$ and also alters HIF-1 $\alpha$ biology, ${ }^{23,35}$ further work is necessary to clarify the causal mechanistic basis of mutant IDH1 biology.

Accumulating evidence suggests that $I D H I$ mutant and IDHI wild-type tumors should perhaps be considered different tumors, albeit with similar histopathology. Patients with $I D H 1$ mutant tumors are nearly 2 decades younger than patients with $I D H I$ wild-type tumors and exhibit an overall survival that can be up to 3 -fold greater than patients with wild-type tumors. ${ }^{28,46}$ This survival advantage is even more amplified in lower-grade astrocytomas. Additionally, IDHI mutant tumors appear to harbor distinct imaging characteristics: they often enhance less with gadolinium contrast, harbor less peritumoral edema, exhibit increased cystic components, and have an increased incidence of frontal lobe involvement. ${ }^{14,37}$ It is likely that the $I D H 1$ mutation is a primordial alteration in tumor development. In recent work from the Costello group, all IDHI mutant low-grade gliomas that recurred still harbored IDHI mutations despite substantial differences in other tumor-associated genes, ${ }^{33}$ suggesting that this alteration occurs in the tumor's cell of origin. Ultimately, these distinct clinical characteristics have raised the possibility that $I D H 1$ status may influence how mutant tumors are treated. Indeed, Beiko et al. showed that resection of total $I D H 1$ mutant malignant astrocytoma volume-i.e., both $\mathrm{T} 1$ enhancing and T2 hyperintense areas-was associated with a median survival longer than 9 years; in comparison, the only survival benefit in IDHI wild-type malignant astrocytomas was associated with resection of T1 enhancing disease. ${ }^{5}$ This study is perhaps the first to demonstrate that an understanding of tumor heterogeneity in glioblastoma is not just a basic science interest but may significantly influence how we surgically manage glioblastomas from patient to patient.

\section{Intratumoral Heterogeneity: Tumors Within Tumors}

The realization that tumors can vary structurally from patient to patient has been complemented by additional recent work demonstrating that there is much more to tumor heterogeneity than we had anticipated. Specifically, recent studies have demonstrated that there is substantial subclonality within each tumor in a manner that suggests that patients harbor glioblastomas that resemble admixtures of molecularly distinct tumors rather than a uniform disease. In particular, studies that have focused on the distribution of alterations of receptor tyrosine kinases have provided a window into the intratumoral diversity seen in glioblastoma. Simply stated, it is becoming clear that glioblastoma is a composite of multiple tumors within the same tumor, and the extent to which we deeply understand this intrapatient heterogeneity will have significant therapeutic implications.

Receptor Tyrosine Kinase Heterogeneity: The Subclonal Landscape. The study of both the activation states and the distribution of SCNA in receptor tyrosine kinases (RTKs) in glioblastoma has provided powerful evidence of the subclonal landscape within these tumors. Receptor tyrosine kinases are highly dysregulated in glioma, with high-level amplification observed in RTKs such as EGFR (60\%-70\%), PDGFRA (12\%-15\%), and MET (5\%). ${ }^{11}$ However, monotherapy with RTK inhibitors has been inefficacious to date in this tumor type. ${ }^{32}$ It has been demonstrated previously that the EGFRvIII variant, which is found in up to $20 \%$ of glioblastomas, ${ }^{11}$ is heterogeneously expressed across EGFRvIII-positive tumors, ${ }^{43}$ suggesting that its true contribution to gliomagenesis stems from paracrine mechanisms. ${ }^{31,43}$ In addition, Stommel et al. demonstrated that RTKs likely cooperate to drive mitogenic signaling in glioblastoma. ${ }^{57}$ Specifically, the majority of glioblastoma cell lines analyzed exhibited coactivation of 3 or more RTKs. Moreover, immunofluorescence staining with phosphospecific antibodies for multiple RTKs revealed variable coexpression of activated RTKs among individual cells derived from a single primary glioblastoma tumor. Thus, there is promiscuous RTK activation within a single glioblastoma tumor, although the etiology for this coactivation state is not known.

Recent work provided further compelling evidence of clonal heterogeneity within a single tumor on the structural genomics level. Both Snuderl and Szerlip and their colleagues discovered that nearly $13 \%$ of glioblastomas carrying amplifications of EGFR, MET, or PDGFRA harbored multiple copy number gains in these genes. ${ }^{54,61}$ Exclusivity analysis revealed that the predominant pattern was mosaic amplification of these RTKs rather than coamplification within a single cell. This mosaic pattern of RTK amplification was graphically illustrated in a remarkable figure in which fluorescence in situ hybridization analysis for EGFR and PDGFRA was performed on a coronal section of a whole brain autopsy specimen from a patient with infiltrative left parietal glioblastoma. Whereas the large left parietal mass harbored EGFRamplified and PDGFRA-amplified cells, the majority of cells within the infiltrated right contralateral tumor were EGFR amplified. These studies therefore provide clear examples of the subclonal architecture in glioblastoma and suggest that there may be a high potential for greater diversity when we begin to explore other molecular features beyond RTK alterations.

Heterogeneity Identified by Analyzing Single Cells. The future of heterogeneity analysis will be conducted at the single cell level. Although TCGA and other genomic studies to date have been incredibly informative, they were conducted on bulk tumor DNA samples and therefore were not able to capture cell-to-cell variability. Two recent studies isolated and analyzed single cells, thereby providing not only proof-of-principle of this type of approach in glioblastoma but important information about the nature of heterogeneity in a single nucleus. It is important to note here that the type of sequencing possible from single cell nucleic acids is not yet at the same high coverage as that performed from larger amounts of DNA, and the types of whole genome amplification necessary to do this kind of analysis can still be prone to error. Nevertheless, this field is developing rapidly. Patel et al. used single-cell RNA sequencing to profile 430 cells from 5 
different primary glioblastomas ${ }^{47}$ and provided a stunning demonstration of intratumoral heterogeneity. Cells from the same tumor expressed different RTKs, and there was a gradient of "stemness" capability from cell to cell within each tumor. Strikingly, using the transcriptomic subtypes from TCGA, ${ }^{11,63}$ each tumor was found to harbor several gene expression subtypes such that several tumors harbored single cells from the mesenchymal, classical, and proneural classes. Moreover, in a clever modified analysis of TCGA survival data, the authors found that, whereas proneural classification was associated with increased overall survival, the extent of "contamination" of the proneural subtype by other subtype signatures significantly reduced survival. Thus, this study provided the first look at the spectrum of biological programs as read out by transcriptional profile within distinct cells in a bulk tumor and strongly suggests that glioblastomas are ensembles of many cells - or cell states - that has translational relevance.

An additional study using single cell analysis focused on DNA, rather than RNA, analysis. Francis et al. isolated nuclei from 2 glioblastomas using flow cytometry and amplified single cell genomes for next-generation sequencing. ${ }^{24}$ Multiple distinct alterations can lead to the coexistence of various EGFR aberrations within tumors, including chromosomal break points, EGFR variants, extracellular domain missense mutations, and carboxylterminal truncation. Among several interesting findings, this study showed that there was heterogeneous expression of oncogenic EGFR variants within single cells of the same tumor, all of which were mutually exclusive, implying that these variants evolved independently in a convergent manner. Further work using the elegant approach described will undoubtedly reveal novel insights into oncogene cooperativity, mechanisms of subclonal evolution, and more detailed analysis of the types of alterations found in single cells, especially as DNA sequencing coverage potential increases.

Taken together, it is becoming clear that, next to invasion, the hallmark of glioblastoma is heterogeneity-both between individual tumors and within each tumor (Fig. 1). Further study on the single cell level will be crucial to understand how subclonal ensembles behave, what the nature of cell state transitions might be, and how populations of subclones can be targeted therapeutically.

\section{Cellular Heterogeneity}

\section{Identification of a Subpopulation of Tumor-Initiating Cells}

Our conceptual framework for tumor biology has been greatly influenced by the introduction of the cancer stem cell hypothesis, which posits a "developmental" tumor hierarchy maintained by a rare fraction of cells with stem cell-like properties called "cancer stem cells" or "tumor-initiating cells" (TICs). ${ }^{36}$ Although the identity of a true "cancer stem cell" in glioblastoma and its contribution to the overall disease process remain somewhat controversial, there has been intensive investigation of glioblastoma cell subpopulations with the biological properties of self-renewal in vitro and tumor formation in xenografts in vivo. In glioblastoma, reasonable human data and very rigorous transgenic mouse data exist, which together support the notion that TICs represent a key and influential component of the cellular heterogeneity observed in glioblastoma and is thought to be responsible for overall tumor growth, therapy resistance, and tumor recurrence. $3,8,15,53$

One of the main reasons for dissent regarding the identity of TICs in glioblastoma is the lack of reliable markers that definitively and prospectively identify glioblastoma cells as TICs. Singh and colleagues originally reported that human brain tumor cells expressing cell surface antigen CD133 were capable of self-renewal in vitro and tumor development in the brains of immunodeficient mice. In contrast, CD133-negative cells engrafted to the injection site but failed to form tumors. ${ }^{53}$ However, others have found that CD133-negative tumors can also initiate aggressive tumors in xenograft models. ${ }^{16}$ Among the markers used for identification of TICs (some not applicable in a prospective manner) are Nestin, SOX2, ID1, CD15, CD44, Integrin alpha 6, and the ability to extrude Hoechst dye (known as side-population analysis). ${ }^{4,7,38,56}$ In fact, part of the issue with identifying a reliable marker for TICs is that more than one type of TIC likely exists in glioblastoma, a concept most recently supported by the observation that TICs grown in sphere culture cluster predominantly into two transcriptomic subtypes-proneural and mesenchymal-with transcriptional parallels to the respective TCGA subtypes. ${ }^{7}$ Importantly, compared with proneural TICs, mesenchymal TICs were relatively radioresistant, in a manner dependent on NF- $\kappa \mathrm{B}$ activity. Moreover, these subtypes themselves were found to be dynamic and plastic, as proneural TICs could be converted into mesenchymal TICs in culture through exposure to tumor necrosis $-\alpha$, a factor elaborated by microglia, suggesting that the microenvironment might be capable of controlling the transcriptional signature and consequently the phenotype of TICs potentially in vivo. Given the discovery of diverse and shifting transcriptional profiles in TICs, it is perhaps not surprising that a consistent and ubiquitous TIC marker has been challenging to identify.

Besides cell extrinsic factors, recent exciting data have highlighted the importance of epigenetic regulation in defining the TIC phenotype. Using global profiling of the histone H3 Lysine27 acetylation (H3K27Ac), which marks genomic loci with active enhancers and promoters, Suvà and colleagues uncovered an epigenetic signature that defines the self-renewal and tumorigenic potential of TICs. ${ }^{60}$ Conceptually similar to work done on induced pluripotent stem cells (iPSCs), this epigenetic profile provided a roadmap to identifying the master regulator transcription factors required for tumor initiation and moreover were sufficient to "reprogram" differentiated and nontumorigenic glioblastoma cells into TIClike cells, which they named "induced tumor-propagating cells" (iTPCs). ${ }^{60}$ The transcription factors identified were POU3F2, SOX2, SALL2, and OLIG2, the last of which could be interchanged with the epigenetic regulator RCOR2. Remarkably, sequential gene "add-back" of the 4 identified transcription factors in differentiated glioblastoma cells recapitulated the $\mathrm{H} 3 \mathrm{~K} 27 \mathrm{Ac}$ epigenetic 


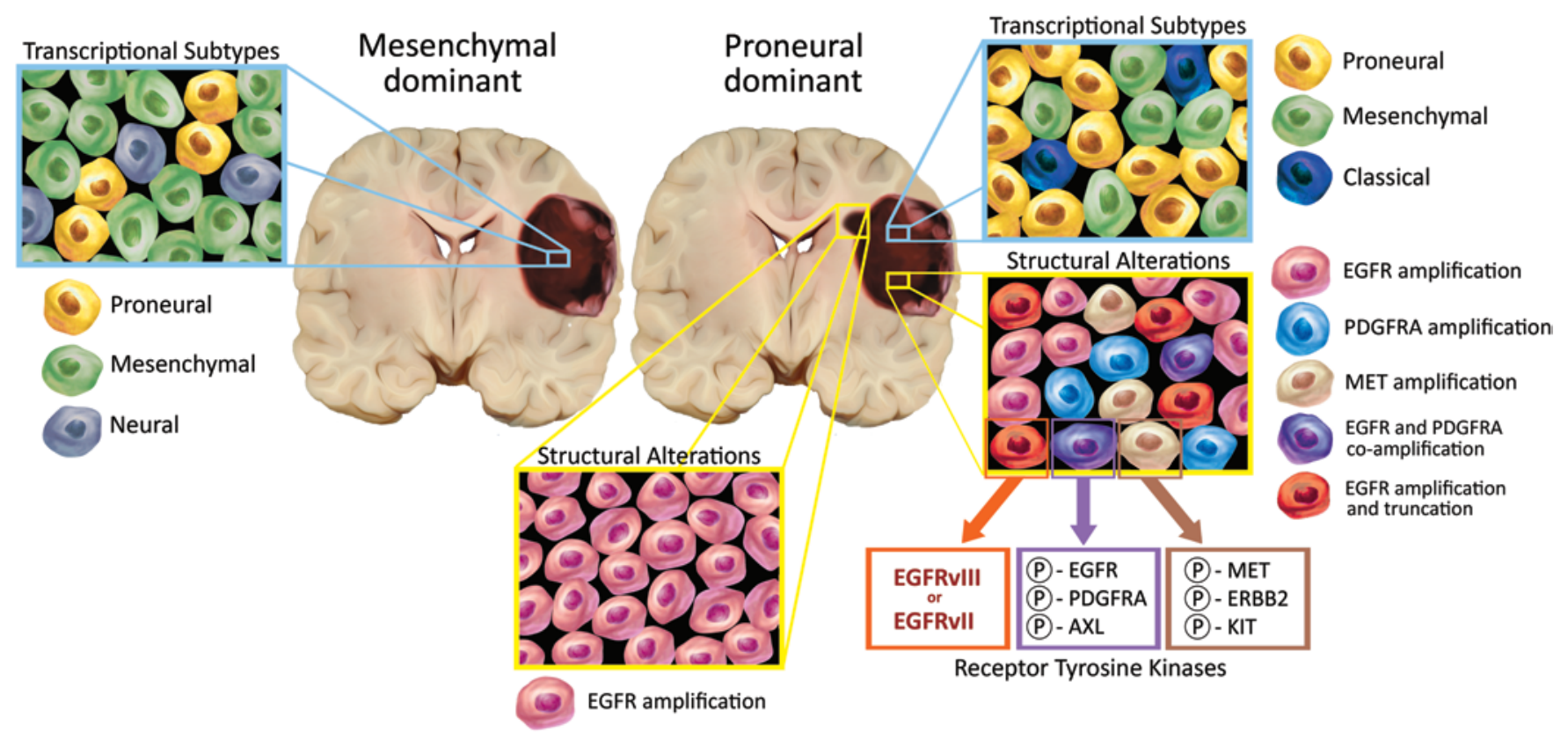

FIG. 1. Schematic diagram of intratumoral heterogeneity. It is likely that each glioblastoma tumor represents a composite of heterogeneous subclones. This diversity can be defined by the pattern of RTK amplification and by transcriptional subtype. Here we depict two left-hemisphere glioblastomas, one Mesenchymal dominant (left panel) and another Proneural dominant (right panel). While both are composed of a mosaic of cellular subclones based on transcriptional profiling, in the Proneural dominant tumor each subclone also harbors distinct, spatially restricted RTK amplifications.

signature observed in TICs, suggesting that these genes in collaboration could sculpt the chromatin state of glioblastoma cells. These results also raise the intriguing idea that an epigenetic profile might represent the underlying abnormality in TICs. Together, it appears that the TIC, rather than representing one particular cell type arising from a particular transformed stem/progenitor cell, more likely represents a dynamic-rather than fixed-cell state in flux, shaped by genetic, epigenetic, and microenvironmental events (Fig. 2).

\section{TIC Involvement in Treatment Resistance and Recurrence}

Glioblastoma remains one of the most lethal human malignancies in part due to resistance to radiation therapy. TICs in glioblastomas are hypothesized to contribute to resistance mechanisms. Bao and colleagues demonstrated that ionizing radiation enriched the TIC subpopulation, as shown through increased CD133 positivity, in culture and in xenografts, likely due to diminished apoptosis. ${ }^{3}$ Mechanistically, the authors suggested that CD133-positive cells exhibited higher phosphorylation and therefore activation of regulatory proteins that respond to DNA damage. TICs have also been implicated in tumor recurrence following treatment with the alkylating agent temozolomide. Although the relative resistance of TICs to temozolomide in comparison with their non-TIC counterparts remains unclear, clever transgenic mouse experiments have clearly demonstrated that TICs contribute to recurrence following temozolomide. ${ }^{15}$ Specifically, Chen and colleagues used a genetic mouse model of glioblastoma with conditional loss of major tumor suppressors PTEN, NF1, and P53 in neural stem cells and crossed these tumor-prone mice with a transgenic mouse that marks Nestin-positive TICs. ${ }^{15}$ Temozolomide eradicated the majority of proliferative cells in tumors in vivo, which was then followed by reemergence of cell division and tumor growth via the Nestin-positive TIC subpopulation. Thus, the distinct responses of TICs versus non-TICs to standard chemoradiation therapies highlights the clinical importance of cellular heterogeneity and motivates further investigation into the mechanisms underlying these divergent treatment responses.

\section{Trans-Differentiation of TICs}

Although it is well established that TICs can undergo aberrant differentiation along restricted neural lineages, emerging evidence suggests that the stem cell-like properties of TICs might also render TICs capable of adopting nonneural fates. Several groups have reported that TICs have the capacity to transdifferentiate into components of vessels, namely endothelial cells and pericytes, suggesting the possibility that glioblastoma tumors may sculpt their own environment. The tumor perivascular niche exhibits striking parallels with the developmental niche seen with normal neural stem/progenitor cells and has been shown to act as a sanctuary for TICs. ${ }^{39}$ Three groups first reported that glioblastoma TICs can transdifferentiate into endothelial cells..$^{51,55,64}$ As a proof of concept that tumor-derived endothelial cells contribute to the growth of glioblastoma tumors, disruption of endothelial cells arising from TICs in a xenograft model reduced tumor volume. ${ }^{51}$ Additionally, Soda and colleagues demonstrated that the development of tumor-derived endothelial cells were resistant to anti-vascular endothelial growth factor treatment in culture and in a glioblastoma mouse model. ${ }^{55}$ Therefore, the presence of tumor-derived 


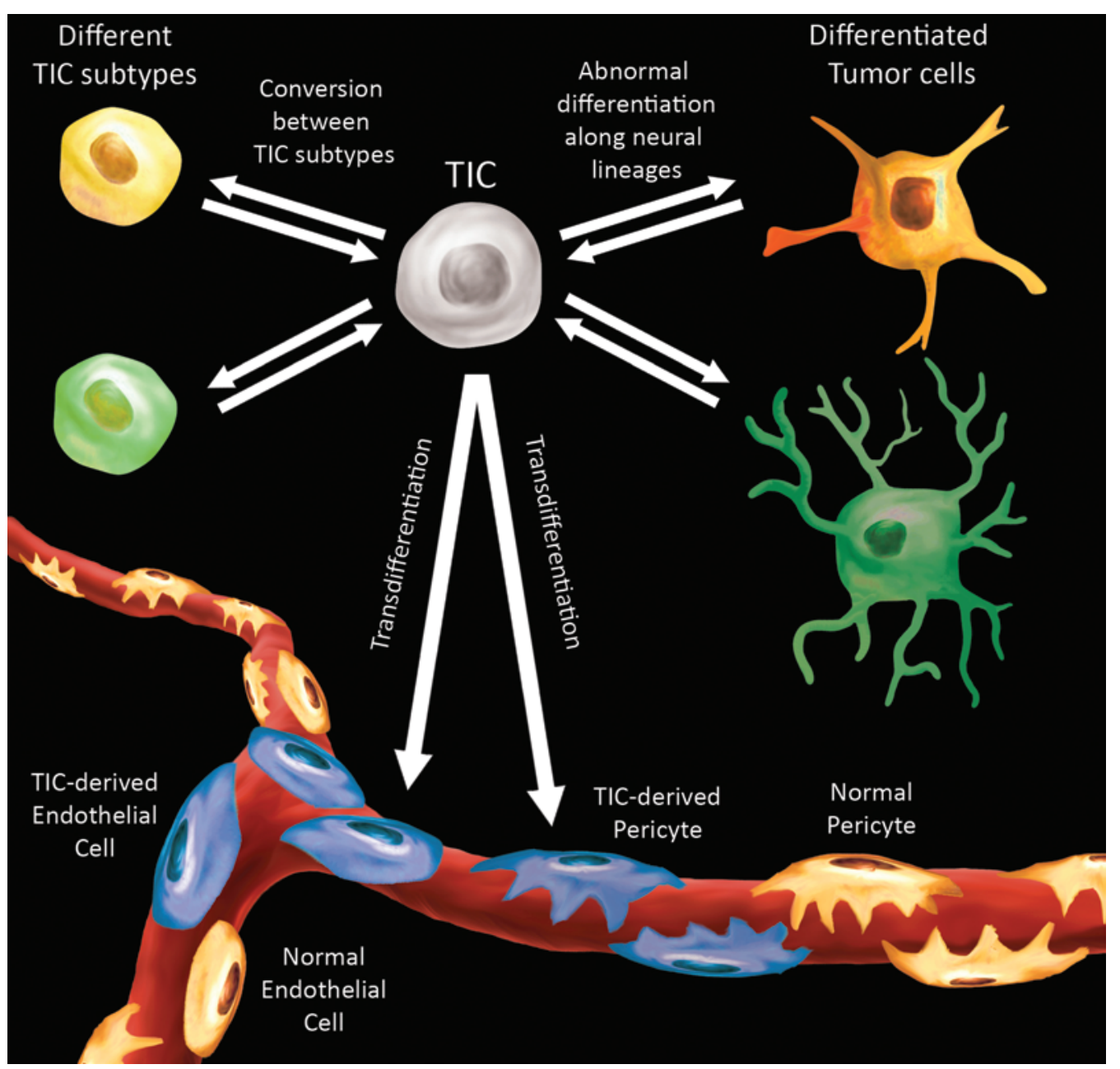

FIG. 2. Tumor-initiating cells (TICs) represent a fluid cell state. Although operationally defined by their ability to self-renew and form tumors in immunocompromised mice, TICs demonstrate tremendous biological diversity, from aberrant differentiation down restricted neural lineages to conversion into alternative TIC subtypes to transdifferentiation along nonneural lineages.

endothelial cells in xenografts and clinical specimens from glioblastoma patients highlights the clinical value of considering TIC transdifferentiation in therapy development. ${ }^{51,55,64}$ Cheng and colleagues used rigorous in vivo cell lineage tracing and a large number of human glioblastoma specimens to demonstrate that TICs can also differentiate into vascular pericytes. ${ }^{17}$ Interestingly, in the pericyte study, the proportion of tumor-derived pericytes in human samples appeared much more significant than that of tumor-derived endothelial cells. Targeting of tumor-derived pericytes disrupted tumor vasculature and impacted tumor growth in xenografts. Together, the cellular heterogeneity provided by transdifferentiation of TICs into specific vascular cells supports the idea that glioblastomas actively establish and remodel a favorable tumor environment.

\section{Discussion}

We have used key examples in the literature to illustrate our growing understanding that heterogeneity-both molecular and cellular-is a defining hallmark of this disease (Table 1). Clearly, heterogeneity analysis is a rapidly expanding field, and with further technological advances in genomic profiling approaches at the single cell level, there will surely be additional insights obtained in a short period of time.

A remaining and intriguing question regarding both molecular and cellular heterogeneity is the relationship between structural alterations and cellular phenotypes within a single tumor. Might certain cellular phenotypes including the TIC state be enriched for particular structural alterations? Similarly, as suggested by the findings of Snuderl and colleagues, ${ }^{54}$ are particular niches or domains within a tumor enriched for distinct mutations that either establish or select for that tumor niche? Moreover, what is the relationship between cell-intrinsic tumor heterogeneity and cells of the brain tumor microenvironment? Finally, are the fixed molecular alterations or biological programs that drive the dynamic and fluid interchange that may occur between cellular phenotypes/cell states/transcriptional subtypes?

We have just scratched the molecular surface of this complex brain cancer and are already confronted with the challenging, if not disturbing, reality that glioblastomas are not only molecularly diverse across different patients 
but that they also consist of multiple molecularly distinct tumors with different cellular phenotypes within one tumor. Our challenge is to understand the principles underlying this massive amount of data on tumor heterogeneity to offer insight into prognosis and guide our management of this devastating disease. The goal of translating these scientific observations into effective clinical practice will undoubtedly continue to require discovery that can only come from rigorous basic science. Indeed, the data suggest that the kind of profiling efforts conducted through TCGA may need to be applied to 1000-10,000 individual cells within a similar number of patient tumors. Ultimately, there is still much to learn about how our emerging appreciation of tumor heterogeneity can be harnessed to predict patient prognosis and direct treatment.

\section{Disclosure}

The authors report no conflict of interest concerning the materials or methods used in this study or the findings specified in this paper.

Author contributions to the study and manuscript preparation include the following. Conception and design: AH Kim, Leuthardt, Dunn. Drafting the article: AH Kim, Aum, DH Kim, Beaumont, Dunn. Critically revising the article: AH Kim, Beaumont, Dunn. Reviewed submitted version of manuscript: all authors. Approved the final version of the manuscript on behalf of all authors: Dunn. Administrative/technical/material support: AH Kim, Aum, Beaumont. Study supervision: Leuthardt.

\section{References}

1. Alizadeh AA, Eisen MB, Davis RE, Ma C, Lossos IS, Rosenwald A, et al: Distinct types of diffuse large B-cell lymphoma identified by gene expression profiling. Nature 403:503-511, 2000

2. Andronesi OC, Rapalino O, Gerstner E, Chi A, Batchelor TT, Cahill DP, et al: Detection of oncogenic IDH1 mutations using magnetic resonance spectroscopy of 2-hydroxyglutarate. J Clin Invest 123:3659-3663, 2013

3. Bao S, Wu Q, McLendon RE, Hao Y, Shi Q, Hjelmeland AB, et al: Glioma stem cells promote radioresistance by preferential activation of the DNA damage response. Nature 444:756-760, 2006

4. Barrett LE, Granot Z, Coker C, Iavarone A, Hambardzumyan D, Holland EC, et al: Self-renewal does not predict tumor growth potential in mouse models of high-grade glioma. Cancer Cell 21:11-24, 2012

5. Beiko J, Suki D, Hess KR, Fox BD, Cheung V, Cabral M, et al: IDH1 mutant malignant astrocytomas are more amenable to surgical resection and have a survival benefit associated with maximal surgical resection. Neuro Oncol 16:81-91, 2014

6. Beroukhim R, Getz G, Nghiemphu L, Barretina J, Hsueh T, Linhart D, et al: Assessing the significance of chromosomal aberrations in cancer: methodology and application to glioma. Proc Natl Acad Sci U S A 104:20007-20012, 2007

7. Bhat KP, Balasubramaniyan V, Vaillant B, Ezhilarasan R, Hummelink K, Hollingsworth F, et al: Mesenchymal differentiation mediated by $\mathrm{NF}-\kappa \mathrm{B}$ promotes radiation resistance in glioblastoma. Cancer Cell 24:331-346, 2013

8. Bleau AM, Hambardzumyan D, Ozawa T, Fomchenko EI, Huse JT, Brennan CW, et al: PTEN/PI3K/Akt pathway regulates the side population phenotype and ABCG2 activity in glioma tumor stem-like cells. Cell Stem Cell 4:226-235, 2009

9. Bredel M, Scholtens DM, Harsh GR, Bredel C, Chandler JP, Renfrow JJ, et al: A network model of a cooperative genetic landscape in brain tumors. JAMA 302:261-275, 2009
10. Brennan C: Genomic profiles of glioma. Curr Neurol Neurosci Rep 11:291-297, 2011

11. Brennan CW, Verhaak RG, McKenna A, Campos B, Noushmehr H, Salama SR, et al: The somatic genomic landscape of glioblastoma. Cell 155:462-477, 2013

12. Campbell PJ, Yachida S, Mudie LJ, Stephens PJ, Pleasance ED, Stebbings LA, et al: The patterns and dynamics of genomic instability in metastatic pancreatic cancer. Nature 467:1109-1113, 2010

13. Cancer Genome Atlas Research Network: Comprehensive genomic characterization defines human glioblastoma genes and core pathways. Nature 455:1061-1068, 2008

14. Carrillo JA, Lai A, Nghiemphu PL, Kim HJ, Phillips HS, Kharbanda S, et al: Relationship between tumor enhancement, edema, IDH1 mutational status, MGMT promoter methylation, and survival in glioblastoma. AJNR Am J Neuroradiol 33:1349-1355, 2012

15. Chen J, Li Y, Yu TS, McKay RM, Burns DK, Kernie SG, et al: A restricted cell population propagates glioblastoma growth after chemotherapy. Nature 488:522-526, 2012

16. Chen R, Nishimura MC, Bumbaca SM, Kharbanda S, Forrest WF, Kasman IM, et al: A hierarchy of self-renewing tumorinitiating cell types in glioblastoma. Cancer Cell 17:362-375, 2010

17. Cheng L, Huang Z, Zhou W, Wu Q, Donnola S, Liu JK, et al: Glioblastoma stem cells generate vascular pericytes to support vessel function and tumor growth. Cell 153:139-152, 2013

18. Chowdhury R, Yeoh KK, Tian YM, Hillringhaus L, Bagg EA, Rose NR, et al: The oncometabolite 2-hydroxyglutarate inhibits histone lysine demethylases. EMBO Rep 12:463-469, 2011

19. Colman H, Zhang L, Sulman EP, McDonald JM, Shooshtari NL, Rivera A, et al: A multigene predictor of outcome in glioblastoma. Neuro Oncol 12:49-57, 2010

20. Dang L, White DW, Gross S, Bennett BD, Bittinger MA, Driggers EM, et al: Cancer-associated IDH1 mutations produce 2-hydroxyglutarate. Nature 462:739-744, 2009

21. Ding L, Ley TJ, Larson DE, Miller CA, Koboldt DC, Welch JS, et al: Clonal evolution in relapsed acute myeloid leukaemia revealed by whole-genome sequencing. Nature 481:506-510, 2012

22. Dunn GP, Andronesi OC, Cahill DP: From genomics to the clinic: biological and translational insights of mutant IDH1/2 in glioma. Neurosurg Focus 34(2):E2, 2013

23. Dunn GP, Rinne ML, Wykosky J, Genovese G, Quayle SN, Dunn IF, et al: Emerging insights into the molecular and cellular basis of glioblastoma. Genes Dev 26:756-784, 2012

24. Francis JM, Zhang CZ, Maire CL, Jung J, Manzo VE, Adalsteinsson VA, et al: EGFR variant heterogeneity in glioblastoma resolved through single-nucleus sequencing. Cancer Discov 4:956-971, 2014

25. Gerlinger M, Rowan AJ, Horswell S, Larkin J, Endesfelder $\mathrm{D}$, Gronroos E, et al: Intratumor heterogeneity and branched evolution revealed by multiregion sequencing. $\mathbf{N}$ Engl J Med 366:883-892, 2012

26. Golub TR, Slonim DK, Tamayo P, Huard C, Gaasenbeek M, Mesirov JP, et al: Molecular classification of cancer: class discovery and class prediction by gene expression monitoring. Science 286:531-537, 1999

27. Grasso CS, Wu YM, Robinson DR, Cao X, Dhanasekaran SM, Khan AP, et al: The mutational landscape of lethal castrationresistant prostate cancer. Nature 487:239-243, 2012

28. Hartmann C, Hentschel B, Wick W, Capper D, Felsberg J, Simon M, et al: Patients with IDH1 wild type anaplastic astrocytomas exhibit worse prognosis than IDH1-mutated glioblastomas, and IDH1 mutation status accounts for the unfavorable prognostic effect of higher age: implications for classification of gliomas. Acta Neuropathol 120:707-718, 2010 
29. Hartmann C, Meyer J, Balss J, Capper D, Mueller W, Christians A, et al: Type and frequency of IDH1 and IDH2 mutations are related to astrocytic and oligodendroglial differentiation and age: a study of 1,010 diffuse gliomas. Acta Neuropathol 118:469-474, 2009

30. Huse JT, Phillips HS, Brennan CW: Molecular subclassification of diffuse gliomas: seeing order in the chaos. Glia 59:1190-1199, 2011

31. Inda MM, Bonavia R, Mukasa A, Narita Y, Sah DW, Vandenberg S, et al: Tumor heterogeneity is an active process maintained by a mutant EGFR-induced cytokine circuit in glioblastoma. Genes Dev 24:1731-1745, 2010

32. Jansen M, de Witt Hamer PC, Witmer AN, Troost D, van Noorden CJ: Current perspectives on antiangiogenesis strategies in the treatment of malignant gliomas. Brain Res Brain Res Rev 45:143-163, 2004

33. Johnson BE, Mazor T, Hong C, Barnes M, Aihara K, McLean $\mathrm{CY}$, et al: Mutational analysis reveals the origin and therapydriven evolution of recurrent glioma. Science 343:189-193, 2014

34. Killela PJ, Reitman ZJ, Jiao Y, Bettegowda C, Agrawal N, Diaz LA Jr, et al: TERT promoter mutations occur frequently in gliomas and a subset of tumors derived from cells with low rates of self-renewal. Proc Natl Acad Sci U S A 110:60216026, 2013

35. Koivunen P, Lee S, Duncan CG, Lopez G, Lu G, Ramkissoon $\mathrm{S}$, et al: Transformation by the (R)-enantiomer of 2-hydroxyglutarate linked to EGLN activation. Nature 483:484-488, 2012

36. Kreso A, Dick JE: Evolution of the cancer stem cell model. Cell Stem Cell 14:275-291, 2014

37. Lai A, Kharbanda S, Pope WB, Tran A, Solis OE, Peale F, et al: Evidence for sequenced molecular evolution of IDH1 mutant glioblastoma from a distinct cell of origin. J Clin Oncol 29:4482-4490, 2011

38. Lathia JD, Gallagher J, Heddleston JM, Wang J, Eyler CE, Macswords J, et al: Integrin alpha 6 regulates glioblastoma stem cells. Cell Stem Cell 6:421-432, 2010

39. Lathia JD, Heddleston JM, Venere M, Rich JN: Deadly teamwork: neural cancer stem cells and the tumor microenvironment. Cell Stem Cell 8:482-485, 2011

40. McDermott U, Downing JR, Stratton MR: Genomics and the continuum of cancer care. N Engl J Med 364:340-350, 2011

41. Mermel CH, Schumacher SE, Hill B, Meyerson ML, Beroukhim R, Getz G: GISTIC2.0 facilitates sensitive and confident localization of the targets of focal somatic copy-number alteration in human cancers. Genome Biol 12:R41, 2011

42. Navin N, Kendall J, Troge J, Andrews P, Rodgers L, McIndoo $\mathrm{J}$, et al: Tumour evolution inferred by single-cell sequencing. Nature 472:90-94, 2011

43. Nishikawa R, Sugiyama T, Narita Y, Furnari F, Cavenee WK, Matsutani M: Immunohistochemical analysis of the mutant epidermal growth factor, deltaEGFR, in glioblastoma. Brain Tumor Pathol 21:53-56, 2004

44. Northcott PA, Jones DT, Kool M, Robinson GW, Gilbertson RJ, Cho YJ, et al: Medulloblastomics: the end of the beginning. Nat Rev Cancer 12:818-834, 2012

45. Ostrom QT, Bauchet L, Davis FG, Deltour I, Fisher JL, Langer CE, et al: The epidemiology of glioma in adults: a "state of the science" review. Neuro Oncol 16:896-913, 2014

46. Parsons DW, Jones S, Zhang X, Lin JC, Leary RJ, Angenendt $P$, et al: An integrated genomic analysis of human glioblastoma multiforme. Science 321:1807-1812, 2008

47. Patel AP, Tirosh I, Trombetta JJ, Shalek AK, Gillespie SM, Wakimoto H, et al: Single-cell RNA-seq highlights intratumoral heterogeneity in primary glioblastoma. Science 344:1396-1401, 2014

48. Patmore HS, Ashman JN, Stafford ND, Berrieman HK, Mac-
Donald A, Greenman J, et al: Genetic analysis of head and neck squamous cell carcinoma using comparative genomic hybridisation identifies specific aberrations associated with laryngeal origin. Cancer Lett 258:55-62, 2007

49. Phillips HS, Kharbanda S, Chen R, Forrest WF, Soriano RH, Wu TD, et al: Molecular subclasses of high-grade glioma predict prognosis, delineate a pattern of disease progression, and resemble stages in neurogenesis. Cancer Cell 9:157-173, 2006

50. Pietrak B, Zhao H, Qi H, Quinn C, Gao E, Boyer JG, et al: A tale of two subunits: how the neomorphic R132H IDH1 mutation enhances production of $\alpha \mathrm{HG}$. Biochemistry 50:48044812, 2011

51. Ricci-Vitiani L, Pallini R, Biffoni M, Todaro M, Invernici G, Cenci T, et al: Tumour vascularization via endothelial differentiation of glioblastoma stem-like cells. Nature 468:824828,2010

52. Riddick G, Fine HA: Integration and analysis of genome-scale data from gliomas. Nat Rev Neurol 7:439-450, 2011

53. Singh SK, Hawkins C, Clarke ID, Squire JA, Bayani J, Hide $\mathrm{T}$, et al: Identification of human brain tumour initiating cells. Nature 432:396-401, 2004

54. Snuderl M, Fazlollahi L, Le LP, Nitta M, Zhelyazkova BH, Davidson CJ, et al: Mosaic amplification of multiple receptor tyrosine kinase genes in glioblastoma. Cancer Cell 20:810817,2011

55. Soda Y, Marumoto T, Friedmann-Morvinski D, Soda M, Liu F, Michiue H, et al: Transdifferentiation of glioblastoma cells into vascular endothelial cells. Proc Natl Acad Sci U S A 108:4274-4280, 2011

56. Son MJ, Woolard K, Nam DH, Lee J, Fine HA: SSEA-1 is an enrichment marker for tumor-initiating cells in human glioblastoma. Cell Stem Cell 4:440-452, 2009

57. Stommel JM, Kimmelman AC, Ying H, Nabioullin R, Ponugoti AH, Wiedemeyer R, et al: Coactivation of receptor tyrosine kinases affects the response of tumor cells to targeted therapies. Science 318:287-290, 2007

58. Stupp R, Hegi ME, Mason WP, van den Bent MJ, Taphoorn MJ, Janzer RC, et al: Effects of radiotherapy with concomitant and adjuvant temozolomide versus radiotherapy alone on survival in glioblastoma in a randomised phase III study: 5-year analysis of the EORTC-NCIC trial. Lancet Oncol 10:459466, 2009

59. Sturm D, Bender S, Jones DT, Lichter P, Grill J, Becher O, et al: Paediatric and adult glioblastoma: multiform (epi)genomic culprits emerge. Nat Rev Cancer 14:92-107, 2014

60. Suvà ML, Rheinbay E, Gillespie SM, Patel AP, Wakimoto $\mathrm{H}$, Rabkin SD, et al: Reconstructing and reprogramming the tumor-propagating potential of glioblastoma stem-like cells. Cell 157:580-594, 2014

61. Szerlip NJ, Pedraza A, Chakravarty D, Azim M, McGuire J, Fang Y, et al: Intratumoral heterogeneity of receptor tyrosine kinases EGFR and PDGFRA amplification in glioblastoma defines subpopulations with distinct growth factor response. Proc Natl Acad Sci U S A 109:3041-3046, 2012

62. Turcan S, Rohle D, Goenka A, Walsh LA, Fang F, Yilmaz E, et al: IDH1 mutation is sufficient to establish the glioma hypermethylator phenotype. Nature 483:479-483, 2012

63. Verhaak RG, Hoadley KA, Purdom E, Wang V, Qi Y, Wilkerson $\mathrm{MD}$, et al: Integrated genomic analysis identifies clinically relevant subtypes of glioblastoma characterized by abnormalities in PDGFRA, IDH1, EGFR, and NF1. Cancer Cell 17:98-110, 2010

64. Wang R, Chadalavada K, Wilshire J, Kowalik U, Hovinga KE, Geber A, et al: Glioblastoma stem-like cells give rise to tumour endothelium. Nature 468:829-833, 2010

65. Wiedemeyer R, Brennan C, Heffernan TP, Xiao Y, Mahoney J, Protopopov A, et al: Feedback circuit among INK4 tumor 
Molecular and cellular heterogeneity in glioblastoma

suppressors constrains human glioblastoma development. Cancer Cell 13:355-364, 2008

66. Wu X, Northcott PA, Dubuc A, Dupuy AJ, Shih DJ, Witt H, et al: Clonal selection drives genetic divergence of metastatic medulloblastoma. Nature 482:529-533, 2012

67. Xu W, Yang H, Liu Y, Yang Y, Wang P, Kim SH, et al: Oncometabolite 2-hydroxyglutarate is a competitive inhibitor of $\alpha$-ketoglutarate-dependent dioxygenases. Cancer Cell 19:1730,2011

68. Yachida S, Jones S, Bozic I, Antal T, Leary R, Fu B, et al: Distant metastasis occurs late during the genetic evolution of pancreatic cancer. Nature 467:1114-1117, 2010

69. Yan H, Parsons DW, Jin G, McLendon R, Rasheed BA, Yuan
W, et al: IDH1 and IDH2 mutations in gliomas. N Engl J Med 360:765-773, 2009

Manuscript submitted August 16, 2014.

Accepted September 22, 2014.

Please include this information when citing this paper: DOI: 10.3171/2014.9.FOCUS14521.

Address correspondence to: Gavin P. Dunn, M.D., Ph.D., Department of Neurological Surgery, Washington University School of Medicine, 660 S. Euclid Ave., St. Louis, MO 63110. email: gpdunn@wustl.edu. 